\title{
Evaluación de la sustentabilidad en organizaciones por medio del Modelo NOP
}

\author{
Assessment of sustainability in organizations through NOP Model
}

Francisco Javier Hernández Ayón

Universidad Autónoma de Nayarit, México

franjha@gmail.com

Hermilio Hernández Ayón

Universidad Autónoma de Nayarit, México

hahermx@hotmail.com

Alicia del Carmen Valencia Ovalle

Universidad Autónoma de Nayarit, México

alvalov11@gmail.com

\section{Resumen}

En la actualidad, las organizaciones enfrentan un gran reto: avanzar hacia el desarrollo sustentable. Existen diferentes enfoques para la evaluación de la sustentabilidad en las organizaciones, sin embargo, ninguno analiza de manera holística y sistémica la sustentabilidad, considerando las cuatro esferas del desarrollo sustentable: ecológica, económica, social y cultural. El presente trabajo de investigación tiene como propósito, desarrollar una metodología holística y sistémica para la evaluación de la sustentabilidad en las organizaciones que parte de la visión del desarrollo sustentable soportado por los aspectos: ecológicos, económicos, sociales y culturales. Las cuatro esferas del desarrollo sustentable, son traducidas e interpretadas en las organizaciones y se desglosan sus componentes, y se utiliza el enfoque en el producto visualizado a lo largo de su ciclo de vida para el análisis de la organización. La Metodología NOP desarrollada, permite comprender cómo, a partir de la interrelación de los subsistemas: Naturaleza, Recursos, 
Factor humano e Ideología y de sus componentes, la evaluación de la sustentabilidad en las organizaciones es posible.

Palabras clave: desarrollo sustentable, organización, producto.

\section{Abstract}

Today, organizations face a major challenge: moving towards sustainable development.

There are different approaches for assessing sustainability in organizations, however, none analyzes holistic and systemic manner sustainability, considering the four areas of sustainable development: ecological, economic, social and cultural. This research aims to develop a holistic and systemic methodology for the assessment of sustainability in organizations that part of the vision of sustainable development supported by aspects: ecological, economic, social and cultural rights. The four areas of sustainable development, are translated and interpreted in organizations and its components are broken down, and the approach is used in the product displayed throughout their life cycle for analysis of the organization. The NOP methodology developed allows us to understand how, from the interrelationship of subsystems: Nature, Resources, Human Factor and Ideology and its components, the assessment of sustainability in organizations is possible.

Key words: sustainable development, organization, product.

Fecha Recepción: Julio 2015 Fecha Aceptación: Diciembre 2015

\section{Introducción}

\section{Los cuatro pilares del desarrollo sustentable}

En 1992, durante la Cumbre de la Tierra de Río de Janeiro, con el objetivo de establecer una alianza mundial nueva y equitativa mediante la creación de nuevos niveles de cooperación entre los Estados, los sectores claves de las sociedades y las personas, se procuró alcanzar acuerdos internacionales en los que se respetaran los intereses de todos y se protegiera la integridad del sistema ambiental y de desarrollo mundial (ONU, 1992). Dichos acuerdos quedaron plasmados en la Declaración de Río sobre el medio ambiente y el desarrollo, y constituyen un reto ineludible para todas las organizaciones de hoy. 
El Principio 1 de dicha Declaración, proclama, que "Los seres humanos constituyen el centro de las preocupaciones relacionadas con el desarrollo sostenible" y el Principio 4 señala, que "A fin de alcanzar el desarrollo sostenible, la protección del medio ambiente deberá constituir parte integrante del proceso de desarrollo y no podrá considerarse de forma aislada" (ONU, 1992). Por lo tanto, no se puede avanzar hacia el desarrollo sustentable sin considerar lo social y lo ambiental de forma integral.

A partir de la Cumbre de la Tierra de Río de Janeiro de 1992, el desarrollo sustentable comprende de manera interdependiente e inseparable, la esfera económica, la social y la ambiental. El Principio 48 del Documento Final de la Cumbre Mundial de la ONU, así lo reconoce: “... los tres componentes del desarrollo sostenible: desarrollo económico, desarrollo social y protección del medio ambiente, como pilares interdependientes que se refuerzan mutuamente" (ONU, 2005). Sin embargo, la Organización de las Naciones Unidas para la Educación, la Ciencia y la Cultura (UNESCO), en la Declaración Universal sobre la Diversidad Cultural realizada en el año 2001, señaló una cuarta condición para lograr el desarrollo sustentable: la diversidad cultural. Al respecto señala: “... la diversidad cultural es tan necesaria para el género humano como la diversidad biológica para los organismos vivos”. Se convierte en “... una de las raíces del desarrollo entendido no sólo en términos de crecimiento económico, sino también como un medio para lograr un balance más satisfactorio intelectual, afectivo, moral y espiritual”. (UNESCO, 2001). Los aspectos económicos, sociales, ambientales y culturales, considerados de manera sistémica e integral, constituyen la esencia del desarrollo sustentable.

\section{Evaluación de la sustentabilidad en las organizaciones}

De acuerdo con la Unión Internacional para la Conservación de la Naturaleza (UICN, 2001), la organización medioambiental global más grande y más antigua del mundo, existen diferentes enfoques para la evaluación de la sustentabilidad en las organizaciones, tales como: indicadores ambientales de la Organización para la Cooperación y el Desarrollo Económicos (OCDE); el Estándar de Diseminación de Datos del Fondo Monetario Internacional (GDDS, por sus siglas en inglés); el enfoque sistémico binario con 
indicadores de personas y ecosistema (como el Wellbeing Assessment y United Nations Environment Programme's Global Envrionmental Outlook, entre otros); el enfoque de tres esferas (economía, sociedad, ambiente) usado por el Banco Mundial, entre otros; el marco de trabajo para el informe de la Agenda 21. Sin embargo, sólo los tres últimos enfoques consideran los aspectos sociales; sólo el segundo considera un enfoque sistémico; y, ninguno analiza de manera holística y sistémica la sustentabilidad, considerando los cuatro pilares del desarrollo sustentable. El presente trabajo de investigación tiene como propósito, desarrollar una metodología holística y sistémica que parte de la visión de las cuatro esferas del desarrollo sustentable: ambiental, económica, social y cultural, para evaluación de la sustentabilidad en las organizaciones.

\section{Características de la Metodología NOP}

La Metodología Naturaleza-Organización-Producto (NOP) es un instrumento para el diagnóstico holístico y sistémico de las organizaciones desde la perspectiva de las cuatro esferas del desarrollo sustentable: ambiental, económica, social y cultural. Estos grandes aspectos del desarrollo sustentable pueden ser visualizados en las organizaciones a través de los siguientes subsistemas y componentes (Tabla 1): 
Tabla 1. Relación entre las esferas del Desarrollo sustentable y los subsistemas de la Metodología NOP.

\begin{tabular}{|c|c|c|}
\hline Desarrollo sustentable & \multicolumn{2}{|c|}{ Metodología NOP } \\
\hline Ambiental & A) Naturaleza & $\begin{array}{ll}\text { 1. } & \text { Atmósfera } \\
\text { 2. } & \text { Hidrósfera } \\
\text { 3. } & \text { Geosfera } \\
\text { 4. } & \text { Biosfera }\end{array}$ \\
\hline Económica & B) Recursos & $\begin{array}{ll}\text { 1. } & \text { Materiales } \\
\text { 2. } & \text { Tecnología } \\
\text { 3. } & \text { Financieros } \\
\text { 4. } & \text { Infraestructura }\end{array}$ \\
\hline Social & C) Factor Humano & $\begin{array}{l}\text { 1. Propietarios y empleados } \\
\text { 2. Clientes y comunidad } \\
\text { 3. Competidores y proveedores } \\
\text { 4. Otras organizaciones externas }\end{array}$ \\
\hline Cultural & D) Ideología & $\begin{array}{l}\text { 1. Misión, visión, valores } \\
\text { 2. Normas, políticas, directrices } \\
\text { 3. Conocimiento } \\
\text { 4. Cosmovisión }\end{array}$ \\
\hline
\end{tabular}

Fuente: Elaboración propia.

El subsistema A) Naturaleza, se refiere a todo lo que forma el universo en cuya creación no ha intervenido el ser humano, esto es: la atmósfera, la hidrósfera, la geosfera y la biosfera (plantas y animales). El subsistema B) Recursos en las organizaciones se relaciona con la "tecnosfera", esto es, con lo creado por el hombre (o artificial) y que tiene una constitución material: materiales, tecnología, recursos financieros, e infraestructura. El subsistema $C$ ) Factor Humano, considera al ser humano en sus roles y relaciones (organizado) y lo contempla de manera individual y/o en sociedad: propietarios y empleados; clientes, comunidad; competidores, proveedores; y, otras organizaciones externas. Finalmente, el subsistema D) Ideología se relaciona con la "noosfera", y se refiere también a lo creado por el hombre (o artificial), pero que no tiene una constitución material en las organizaciones: misión, visión, valores; normas, políticas, directrices; conocimiento; y, cosmovisión. 


\section{El producto como hilo conductor}

La Metodología NOP, se basa en el producto $^{1}$ de una organización visualizado a lo largo de su ciclo de vida, esto es, a lo largo de las etapas de: extracción, transporte, fabricación, uso y eliminación o desecho, lo que comúnmente se conoce como "de la cuna a la tumba". Existen dos dimensiones básicas definidas por el ciclo de vida de un producto: la primera, es la que relaciona de manera longitudinal las diferentes organizaciones que participan en la elaboración del producto y las diferentes etapas de su ciclo de vida; la segunda, se da en sentido transversal en cada etapa, misma que está condicionada por la relación entre el producto y la propia organización. El producto se convierte en el hilo conductor que recoge el esfuerzo transformador de las diferentes organizaciones que lo tocan y en el vínculo entre ellas, por lo que, el análisis de las organizaciones puede llevarse a cabo a través del estudio del producto, visualizado a lo largo de su ciclo de vida (Figura 1).

Figura 1. Dimensión longitudinal y transversal del producto.

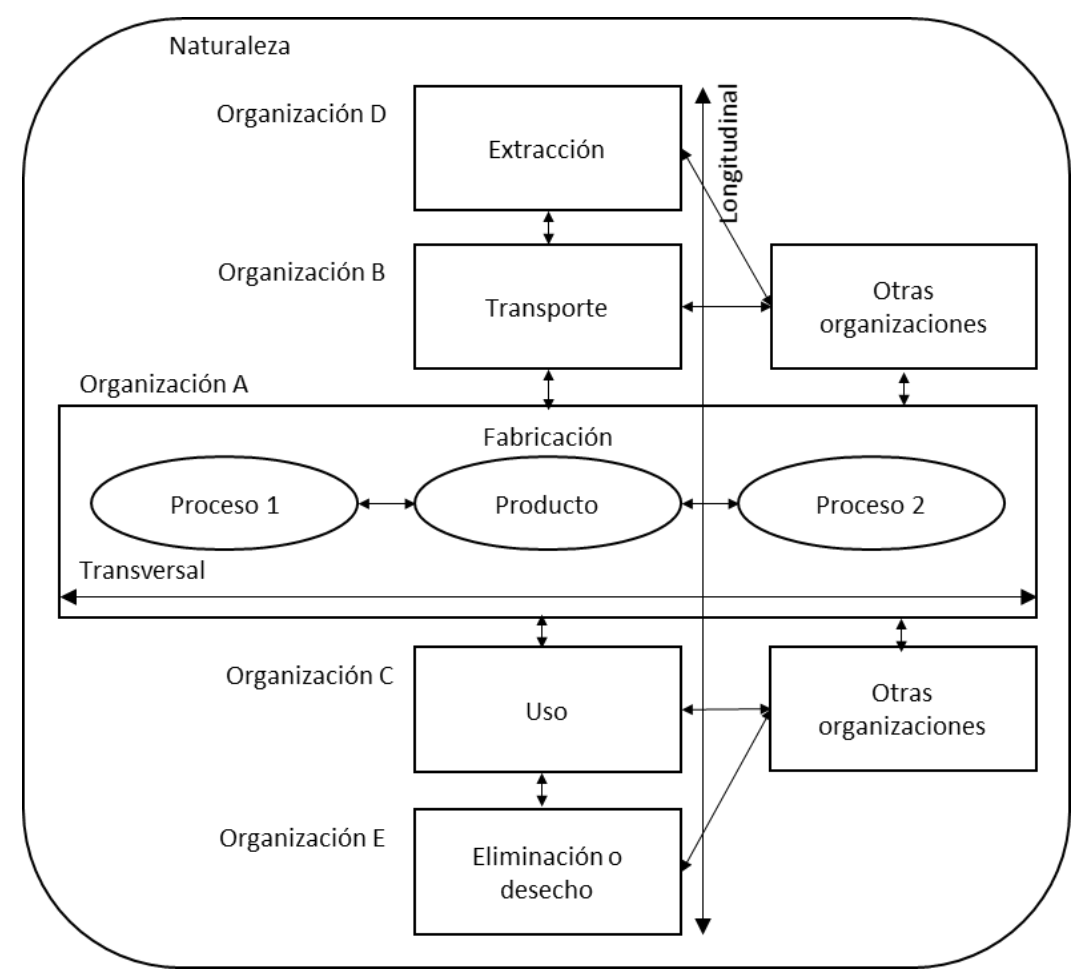

Fuente: Elaboración propia.

\footnotetext{
${ }^{1}$ El producto, en el sentido más amplio de su definición, se refiere tanto a bienes como a servicios.
} 
El producto es el resultado del esfuerzo creador de una organización, a través del cual, ésta cumple su cometido de satisfacer necesidades. Hay una relación directamente proporcional entre esfuerzo creador de una organización y producto. En el producto se transforma y materializa la cultura ${ }^{2}$ de la organización que lo crea; es la síntesis en que se traduce la organización. Al respecto, López et al (1998), a través de su modelo Naturaleza-HombreCultura-Objetos (NHCO), explica, cómo el hombre crea cultura y cómo ésta modela hombres; cómo los objetos - productos culturales- promueven comportamientos y cómo los comportamientos promueven a su vez, la creación de objetos. Este es un ciclo autopoietico que se repite indefinidamente, modificando el entorno inmediato del hombre y por ende su cultura, plasmando en los objetos su manera de pensar, de sentir, sus creencias y su propia esencia. En el mismo sentido, T. Gaudín (citado por Quarante, 1992) señala: "Por una parte la sociedad produce sus objetos, y por la otra, los objetos transforman la sociedad y sus costumbres...".

Los objetos ${ }^{3}$ que produce el hombre tienen dos esencias: una objetiva y otra subjetiva, una derivada de la función y otra del significado, la que procede de la técnica y la que procede del arte. Los objetos, como productos culturales, tienen la capacidad de transmitir información relacionada con estas dos esencias. En este sentido Luengo (2008, p. 324) señala:

"Todo objeto cultural se nos presenta fisicamente bajo una apariencia sensible (palabras, imágenes, sonidos) la cual permite representar algo (acciones, situaciones, lugares). Además, los objetos de la cultura incorporan una serie de componentes metafísicos (ideas, valores, principios) que interpelan al conocimiento

\footnotetext{
${ }^{2}$ La materialización cultural, se da en todos los ámbitos del hombre y es de naturaleza tangible e intangible, son las expresiones culturales que la Organización de las Naciones Unidas para la Educación, la Ciencia y la Cultura (UNESCO) define como: "las expresiones resultantes de la creatividad de las personas, grupos y sociedades, que poseen un conocimiento cultural". Constituyen expresiones culturales el idioma y creencias y hasta los más pequeños y simples objetos, los cuales materializan la cultura de la sociedad y permiten su identificación.

${ }^{3}$ De acuerdo con Ortiz y Espinoza (sin fecha): "los objetos culturales pueden ser de carácter tangible como las herramientas o cualquier otro artefacto inventado por el hombre; de carácter intangible como es el caso de las creencias o concepciones que el ser humano tiene de su universo, de las cuales derivan los mitos y leyendas; y elementos de naturaleza aplicable a la vida social como pueden ser, por ejemplo, las formas de comportamiento y las leyes y normas que las gobiernan, los hábitos, así como las instituciones creadas por el ser humano".
} 
de productores y público. En esta doble dimensión de los objetos culturales, material e ideal, se asienta el carácter simbólico del objeto cultural. Además de significar lo fáctico, un producto cultural puede incorporar una serie de componentes inmateriales, ideas, valores o afectos que interpelan al conocimiento de quienes se hallan implicados en su producción y consumo”.

Reforzando la idea, Restrepo (2000) comenta: “Los objetos tienen funciones, pero también comunican información. Siempre hay un sentido que sobrepasa la función del objeto. Estos sentidos son independientes de la función del objeto porque sólo pueden ser asociados a significados, y las funciones sólo pueden ser asociadas a significantes”.

\section{Metodología NOP}

De manera general, la Metodología NOP se resume en los 3 pasos siguientes: 1) Objetivo y alcance, 2) Análisis de interrelaciones, y 3) Evaluación.

El Objetivo y alcance, consiste en identificar: él o los aspectos que se quieren diagnosticar en la organización en estudio: competitividad, clima organizacional, etc.; los límites del sistema, es decir, la o las etapas del ciclo de vida que considerará el análisis y si se considerará el producto en sus aspectos funcionales, simbólicos o ambos; la unidad funcional, es decir, una medida del producto que servirá de base para el análisis.

El Análisis de las interrelaciones, tiene que ver con el Modelo NOP. A partir de los subsistemas mencionados anteriormente: Naturaleza, Recursos, Factor Humano e Ideología y bajo la doble consideración del producto como hilo conductor y objeto cultural a la vez, se realiza el siguiente Modelo teórico del que parte la Metodología NOP (Figura 2). 
Figura 2. Modelo NOP.
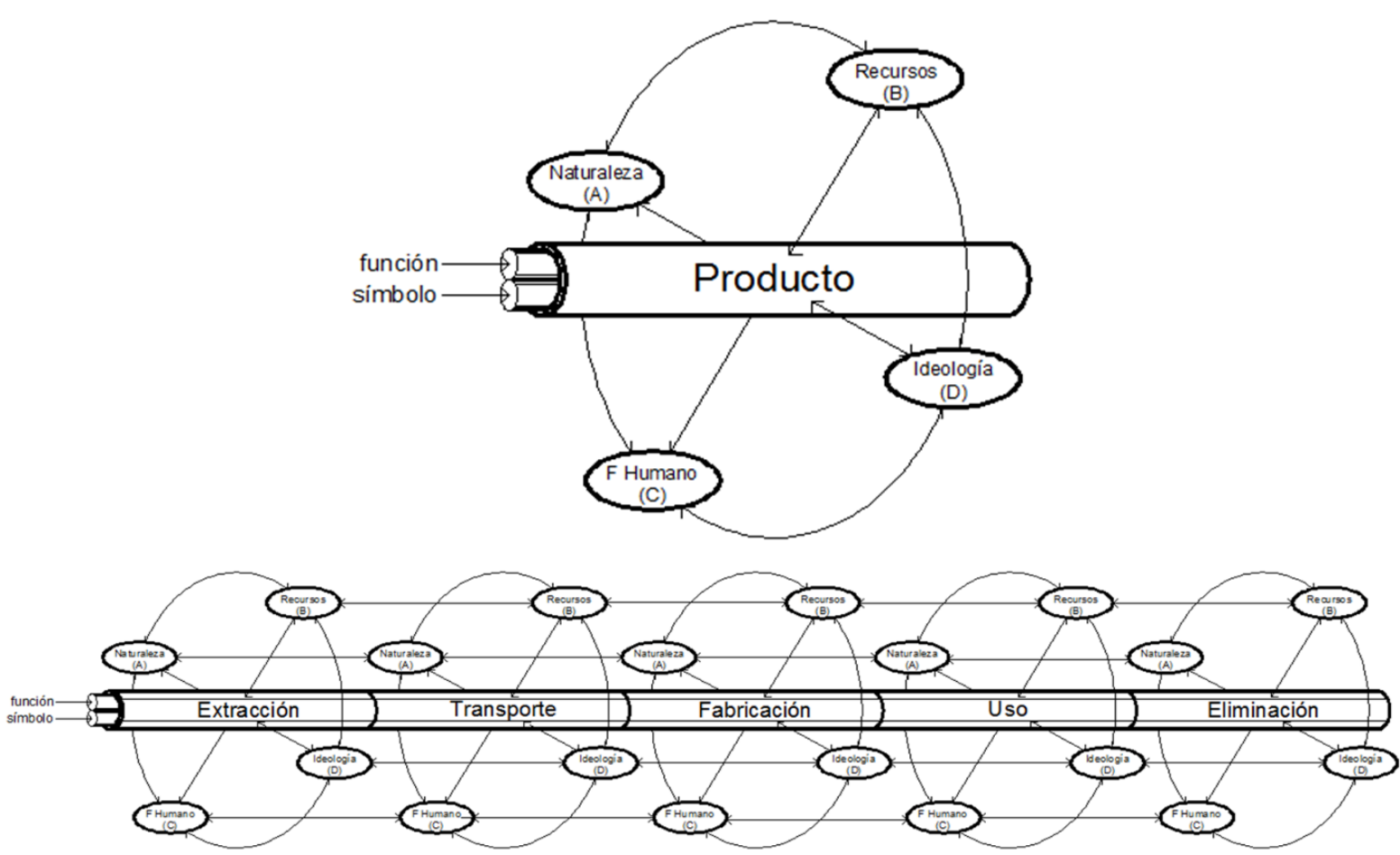

Fuente: Elaboración propia.

Los cuatro subsistemas y componentes que propone la Metodología NOP, forman un sistema complejo de interrelaciones que interactúan y condicionan al producto de una organización. La materialización de los subsistemas en el producto, puede ser explicada por medio de la red de interacciones complejas que surgen de los subsistemas: Naturaleza (A), Recursos (B), Factor Humano $(C)$, Ideología (D) y del producto: 6 para función y 6 para significado (Tabla 2).

Tabla 2. Red de interacciones entre subsistemas, según el Modelo NOP

\begin{tabular}{c|c}
\hline función & significado \\
\hline A-B & A-B \\
\hline A-C & A-C \\
\hline A-D & A-D \\
\hline B-C & B-C \\
\hline B-D & B-D \\
\hline C-D & C-D \\
\hline
\end{tabular}

Fuente: Elaboración propia. 
A nivel de componentes, dichas interrelaciones se convierten a 96 para función y 96 para significado (Tabla 3). La interrelación A1B1, se refiere al análisis del primer componente del subsistema Naturaleza y el primer componente del subsistema Recursos, es decir: atmósfera y materiales; la interrelación A1B2, se refiere al análisis del primer componente del subsistema Naturaleza y el segundo componente del subsistema Recursos, es decir: atmósfera y tecnología; A1B3, se refiere a la interrelación entre atmósfera y lo financiero; A1B4, se refiere a atmósfera y la infraestructura. Esto se realiza para todos los componentes mencionados y entre todos los subsistemas (Tabla 1 y 3 ). El análisis de cada interrelación consiste, en la descripción positiva y/o negativa de dicha relación respecto al problema (aspecto) planteado en el Objetivo y alcance del estudio. Este paso asegura el análisis holístico y sistémico del problema en una organización.

Tabla 3. Interrelaciones entre subsistemas a nivel de componentes

\begin{tabular}{|c|c|c|c|c|c|c|c|c|c|c|c|c|c|c|c|c|c|c|c|c|c|c|c|}
\hline A1 & B1 & $\mathrm{A} 2$ & B1 & A3 & B1 & A4 & B1 & 11 & $\mathrm{C} 1$ & A2 & $\mathrm{C} 1$ & A3 & $\mathrm{C} 1$ & A4 & C1 & A1 & D1 & A2 & D1 & A3 & $\mathrm{D} 1$ & $\mathrm{~A} 4$ & D1 \\
\hline A1 & B & $A 2$ & B2 & A3 & B2 & A4 & B2 & 1 & $C 2$ & 42 & $\mathrm{C} 2$ & A3 & $C 2$ & A4 & $\mathrm{C} 2$ & $\mathrm{~A} 1$ & D2 & A2 & $\mathrm{D} 2$ & $\mathrm{~A} 3$ & $\mathrm{D} 2$ & $\mathrm{~A} 4$ & $\mathrm{D} 2$ \\
\hline $\mathrm{A} 1$ & B3 & $A 2$ & B3 & A3 & B3 & A4 & B3 & A1 & C3 & $\mathrm{A} 2$ & C3 & A3 & C3 & A4 & C3 & $\mathrm{A} 1$ & D3 & A2 & D3 & $\mathrm{A} 3$ & D3 & A4 & D3 \\
\hline A1 & B4 & A2 & B4 & A3 & B4 & A4 & B4 & A1 & $\mathrm{C} 4$ & A2 & C4 & A3 & C4 & A4 & C4 & A1 & D4 & A2 & D4 & A3 & D4 & A4 & D4 \\
\hline & & & & & & & & & & & & & & & & & & & & & & & \\
\hline B1 & C1 & B2 & C1 & B3 & C1 & B4 & C1 & B1 & D1 & B2 & D1 & B3 & D1 & B4 & D1 & C1 & D1 & C2 & D1 & $\mathrm{C} 3$ & D1 & C4 & D1 \\
\hline B1 & $\mathrm{C} 2$ & B2 & $\mathrm{C} 2$ & B3 & C2 & B4 & $\mathrm{C2}$ & B1 & D2 & B2 & $\mathrm{D} 2$ & B3 & D2 & B4 & D2 & C1 & D2 & C2 & D2 & C3 & D2 & C4 & D2 \\
\hline B1 & C3 & B2 & C3 & B3 & C3 & B4 & C3 & B1 & D3 & B2 & D3 & B3 & D3 & B4 & $D$ & C1 & D3 & $C 2$ & D3 & C3 & D3 & C4 & D3 \\
\hline B1 & C4 & B2 & C4 & B3 & C4 & B4 & C4 & B1 & D4 & B2 & D4 & B3 & D4 & B4 & D & C1 & D4 & C2 & D4 & C3 & D4 & C4 & D \\
\hline
\end{tabular}

Fuente: Elaboración propia.

La Evaluación, es un proceso de análisis, síntesis e interpretación de la información obtenida que se basa en la matriz FODA (Fortalezas - Oportunidades - Debilidades Amenazas). Permite identificar y clasificar aquellos factores clave de la organización que pueden influir en la elaboración de estrategias ofensivas, defensivas, adaptativas y de sobrevivencia en la organización.

\section{Conclusiones}

A la luz del desarrollo sustentable, considerado en sus cuatro esferas: ambiental, económica, social y cultural, y enfocándose para su análisis, en el producto, visualizado a lo largo de su ciclo de vida, la Metodología NOP permite el estudio holístico y sistémico de las organizaciones. 
El Modelo NOP, permite comprender cómo, a partir de la interrelación de los subsistemas: Naturaleza, Recursos, Factor humano e Ideología, función y significado, toman una existencia material en el producto -entendido éste en su sentido más amplio- de una organización. Si el producto en una organización materializa la cultura de su sociedad, el conocimiento de la esencia de dicho producto será posible si logramos identificar la manera en que los cuatro subsistemas, toman una existencia material en él. Así, la materialización de la cultura en el producto de una organización, puede ser explicada por medio de la red de interacciones complejas que surgen de Naturaleza, Recursos, Factor Humano, Ideología y del producto, en su aspecto funcional y simbólico. 


\section{Bibliografía}

Luengo, M. (2008). El producto cultural: claves epistemológicas de su estudio. Revista Zer, Vol. 13, Número 24, pp. 317-335. Disponible en: http://www.ehu.es/zer/hemeroteca/pdfs/zer24-15-luengo.pdf

López et al (1998). Propuesta de Investigación: Naturaleza-Hombre-Cultura-Objetos a través del Tiempo. Aproximación a la complejidad del diseño. Revista Theomai. Disponible en: http://theomai.unq.edu.ar/proyrestrepo001.htm

Ortiz, J. y M. Espinoza (Sin fecha). La cultura organizacional y su estudio. Revista de la Universidad Veracruzana. Disponible en: http://colaboracion.uv.mx/default.aspx

ONU (1992). Declaración de Río sobre el medio ambiente y el desarrollo. División de Desarrollo Sostenible, ONU. Disponible http://www.un.org/spanish/esa/sustdev/agenda21/riodeclaration.htm

ONU (2005). Documento final de la cumbre mundial 2005. ONU. Disponible en: http://www.cinu.org.mx/cumbre2005/sala_prensa.htm

Quarante, D. (1992). Diseño Industrial. Elementos introductorios. Barcelona: CEAC.

Restrepo, J. (2000). Diseño-Sociedad-Naturaleza: Hacia un Desarrollo Sostenible en ONU, Latinoamérica, Revista THEOMAI, Número 1. Disponible en: http://revistatheomai.unq.edu.ar/numero1/artrestrepo1.htm

UICN (2001). Herramientas para la evaluación de la sostenibilidad, Parte A. Visión general.

UICN.

Disponible

en: http://cmsdata.iucn.org/downloads/resource_kit_a_esp.pdf 
UNESCO (2001). Declaración Universal de la UNESCO sobre la Diversidad Cultural. UNESCO. $\quad$ Disponible en: http://portal.unesco.org/es/ev.phpURL_ID=13179\&URL_DO=DO_TOPIC\&URL_SECTION=201.html 